\title{
In Situ TEM Study of Order-Disorder Transition in Murataite Ceramics
}

\author{
J. Lian, L. M. Wang, and R. C. Ewing
}

Dept. of Nuclear Engineering \& Radiological Sciences, University of Michigan, Ann Arbor, MI 48109-2104

Murataite is an isometric derivative of the fluorite structure with the general formula $\mathrm{A}_{4} \mathrm{~B}_{2} \mathrm{C}_{7} \mathrm{O}_{22-\mathrm{x}}$ $(0 \leq x \leq 1)$, where eight-coordinated A-sites are occupied by large $\mathrm{Na}^{+}, \mathrm{Ca}^{2+}, \mathrm{REE}^{3+}, \mathrm{An}^{3+/ 4+}$ cations; four-and five-coordinated B-sites, $\mathrm{Mn}^{2+/ 3+}, \mathrm{Zn}^{2+}$ cations; six-coordinated C-sites, small $\mathrm{Ti}^{4+}, \mathrm{Fe}^{3+}$, $\mathrm{Al}^{3+}$ cations. Depending on synthesis conditions, these cations have a great tendency for ordering into specific sites that lead to an ordered murataite structure with varying multiples of the fluorite unit cell. Murataite can occur as a superstructure of 3 multiple (Mu-3C), 5 multiple (Mu-5C) and 8 multiple (Mu-8C) of the fluorite derivative structure, as shown in Fig. 1. These ordered murataite structure-types are closely related to pyrochlore, which has two multiples of the fluorite unit cell. As reported earlier, an order-disorder structural transformation occurs in pyrochlore caused by ion irradiation-induced cation disordering, and this structural transition has an important effect on the materials response to ion irradiation damage. [1,2] These various murataite structure-types provide a great opportunity to understand the influence of the extent of ordering on materials radiation resistance by examining ion beam damage effects in murataite ceramics.

Fig. 2 shows the ex-situ HRTEM images of Mu-5C irradiated by $1 \mathrm{MeV} \mathrm{Kr}^{+}$at various doses at room temperature. Similar to pyrochlore, an ion irradiation-induced order-disorder structural transformation occurs in murataite, as evidenced by the disappearance of supperlattice in the ordered murataite (Figs. 2A and 2C). This structural transformation occurs simultaneously with the amorphization process (Figs. 2B and 2C). An in-situ TEM observation was conducted in order to examine the details of the structural transition. As shown in Fig. 3, in Mu-5 C, with an increase of ion dose, the diffraction maxima from the superstructure (weak spots in SAED patterns) disappeared gradually, and this ion irradiation-induced order-disorder transition follows the sequence of Mu-5C $\rightarrow \mathrm{Mu} 3 \mathrm{C} \rightarrow$ disordered fluorite $\rightarrow$ amorphous state. A similar transition trend was also observed for $\mathrm{Mu}-8 \mathrm{C}$. For $\mathrm{Mu}-5 \mathrm{C}$, this ion irradiation-induced disordered fluorite is unstable relative to amorphous state under $1 \mathrm{MeV} \mathrm{Kr}{ }^{+}$irradiation at $750{ }^{\circ} \mathrm{C}$. Fig. 4 shows a sequence of SAED patterns of murataite (without superstructure) under $1 \mathrm{MeV} \mathrm{Kr}{ }^{+}$irradiation at $600{ }^{\circ} \mathrm{C}$. With the increase of ion dose, the relative intensity of the diffraction maxima from the basic fluorite unit cell increases; at a dose of $4.38 \times 10^{14}$ ions $/ \mathrm{cm}^{2}$, a disordered fluorite can be achieved. In contrast to $\mathrm{Mu}-5 \mathrm{C}$ and $\mathrm{Mu}-8 \mathrm{C}$, this disordered fluorite is stable with respect to completely amorphous state at $600{ }^{\circ} \mathrm{C}$. Combining results on murataite with those pyrochlore and fluorite, [3] a generally increasing trend in the susceptibility to ion beam damage is found in the fluorite-related structures as a function of the increasing number of multiples of the fluorite unit cell and the extent of structural ordering. These results provide insight into the design and selection of materials for applications in high radiation environments.

\section{References}

[1] S. X. Wang, et al., Nucl. Instrum. Meth. B 148, (1999) 704.

[2] J. Lian, et al., Phys. Rev. Lett. 87, (2001) 145901.

[3] S. X. Wang, et al., J. Mater. Res. 14, (1999) 4470. 


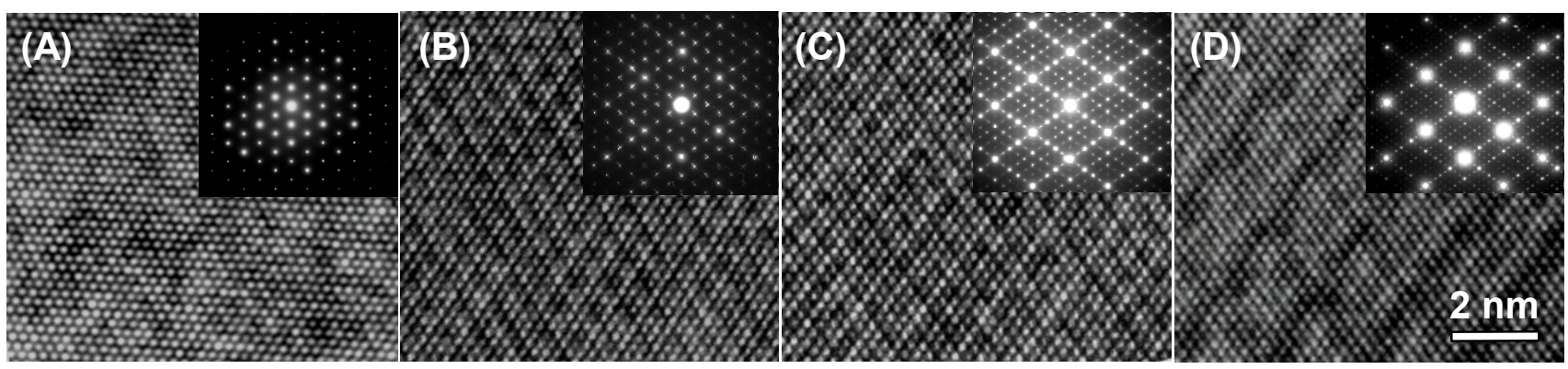

Fig. 1. HRTEM images and SAED patterns showing structural ordering in murataite: (A) without superstructure; (B) 3 multiple; (C) 5 multiple; and (D) 8 multiple of the fluorite unit cell.
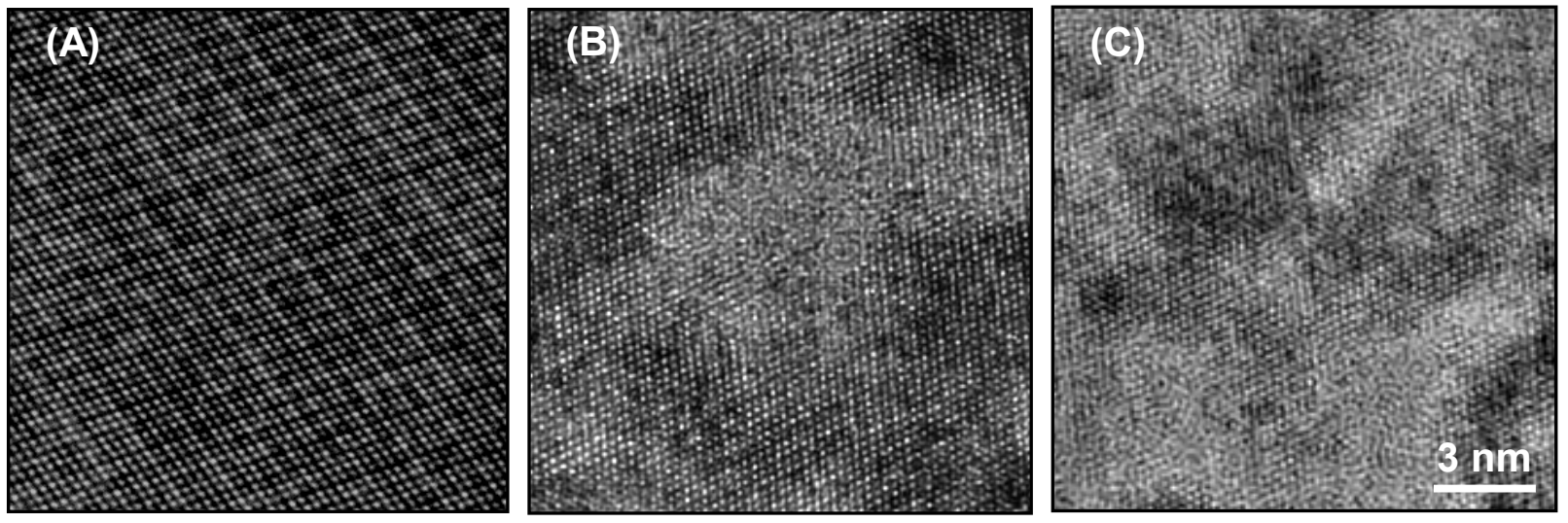

Fig. 2. Ex-situ HRTEM images showing the order-disorder transition in Mu-5C subjected to $1 \mathrm{MeV}$ $\mathrm{Kr}^{+}$irradiation at room temperature: (A) original; (B) 4.38 ; (C) $9.39 \times 10^{13}$ ions $/ \mathrm{cm}^{2}$.
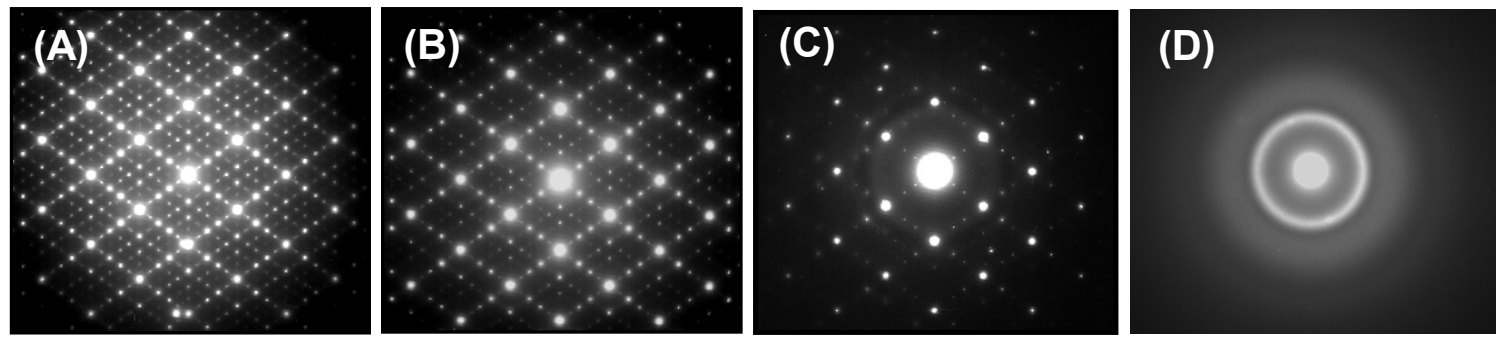

Fig. 3. A sequence of SAED patterns of $\mathrm{Mu}-5 \mathrm{C}$ subjected to $1 \mathrm{MeV} \mathrm{Kr}^{+}$irradiation at $750{ }^{\circ} \mathrm{C}$ : (A) original; (B) 1.25; (C) 3.125; (D) $5.625 \times 10^{14}$ ions $/ \mathrm{cm}^{2}$. An ion irradiation-induced order-disorder transition occurred in murataite from $\mathrm{Mu}-5 \mathrm{C} \rightarrow \mathrm{Mu}-3 \mathrm{C} \rightarrow$ disordered fluorite $\rightarrow$ amorphous state.
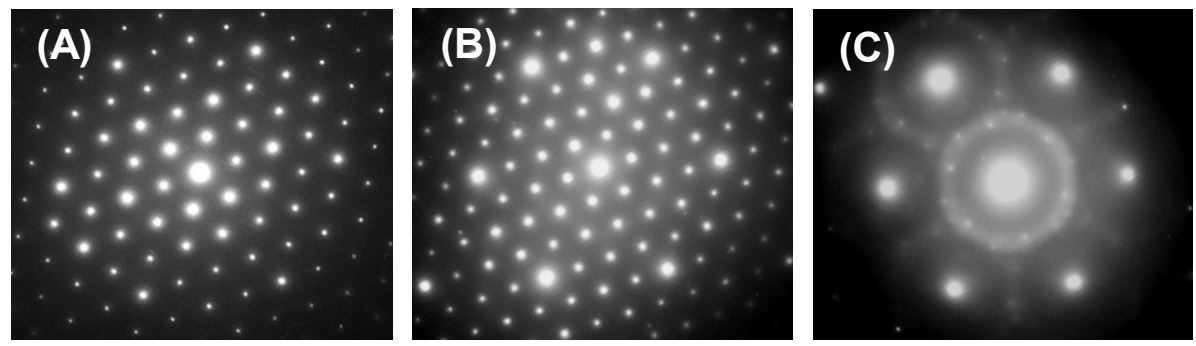

Fig. 4. Ion irradiation-induced murataite-to-fluorite structural transition is revealed by SAD patterns of murataite (without superstructure) under $1 \mathrm{MeV} \mathrm{Kr}^{+}$irradiation at $600{ }^{\circ} \mathrm{C}$ : (A) original; (B) 1.25; (C) $4.38 \times 10^{14}$ ions $/ \mathrm{cm}^{2}$. Disordered fluorite is stable relative to the amorphous state. 